\title{
Size and Charge Stability of Oil Bodies from Peanut
}

\author{
Lihua Hao, ${ }^{1,2}$ Fusheng Chen, ${ }^{1,2}$ Yimiao Xia, ${ }^{1,2}$ Lifen Zhang, ${ }^{1}$ and Ying Xin ${ }^{1}$ \\ ${ }^{1}$ College of Food Science and Technology, Henan University of Technology, No. 100 Lian Hua Rd., Zhengzhou, Henan 450001, China \\ ${ }^{2}$ Collaborative Innovation Center of Grain Storage Security in Henan Province, Zhengzhou, China \\ Correspondence should be addressed to Fusheng Chen; fushengc@haut.edu.cn
}

Received 1 November 2016; Revised 29 November 2016; Accepted 30 November 2016

Academic Editor: Alexandre Giuliani

Copyright (C) 2016 Lihua Hao et al. This is an open access article distributed under the Creative Commons Attribution License, which permits unrestricted use, distribution, and reproduction in any medium, provided the original work is properly cited.

\begin{abstract}
In order to offer scientific bases for the application of oil bodies from peanut in food, this research was undertaken to study the size and charge stability of oil bodies from five peanut varieties. It showed that the mean diameter of oil bodies from yuhua9719 and yuhua9830 is obviously larger than yuhua23, yuhua27, and yuhua9502 in the peanut cell. Moreover, the analysis of diameter distribution of oil bodies also showed that the median diameter of oil bodies increased dramatically in the order of yuhua9719 > yuhua9830 > yuhua23 > yuhua27 > yuhua9502 after aqueous extraction. The charge stability of oil bodies from peanut was observed with zeta $(\zeta)$ potential measurements, which indicated that charge properties and the absolute value of oil bodies from five peanut varieties were significantly affected by $\mathrm{pH}$ and salt concentration, but the degree of influence is different. Of the five peanut varieties, yuhua27 and yuhua9830 possessed excellent charge stability $(\zeta$-potential $>35 \mathrm{mV})$ in neutral microenvironment without salt concentration.
\end{abstract}

\section{Introduction}

Oleaginous plants store lipids in the form of distinct, spherical organelles called oil bodies (oleosomes or spherosomes) that serve as energy stores to support active metabolism [1]. Each oil body has a neutral, lipid matrix core that is comprised mainly of triacylglycerols, and the matrix core is coated by one monolayer of phospholipids embedded with intrinsic proteins [1]. Oil bodies could exist as separate units and serve as an emulsifying agent in a wide variety of products, which range from imitation milk and yogurt to ice cream $[2,3]$. Numerous studies have described the extraction, isolation, and characterization of oil bodies from various plant materials $[4,5]$.

The morphological characteristics of oil bodies, such as size and shape, are species-dependent; these characteristics are affected usually by nutritional and environmental factors $[6,7]$. Oil bodies in different oleaginous plants vary in size from nanoscale to a few $\mu \mathrm{m}[8,9]$. For instance, similar diameters have been observed $(1.45 \mu \mathrm{m})$ in maize and sunflower, and the average size of peanut oil bodies is close to $2 \mu \mathrm{m}[7,10]$. Moreover, the intraspecies differences of oil bodies in different varieties of coffee have shown that $C$. arabica had a different size distribution [11]. In addition, a number of researches have been conducted, which mainly find some relations between contrasted oil extractability and the structural organization of oil bodies (OBs) between different varieties $[12,13]$.

In general, the stability of oil bodies is crucial to the effectiveness of its commercial application in food manufacturing. Previous studies have investigated the influence that factors such as $\mathrm{pH}$, ionic strength, and thermal processing had on the stability of oil bodies that were extracted from different oilseeds. For instance, oil bodies that were extracted from soybeans were stable to aggregation in only a narrow range of $\mathrm{pH}$ values $(\mathrm{pH}=2$ and $\mathrm{pH} \geq 6)$ and $\mathrm{NaCl}$ concentrations $(\leq 25 \mathrm{mM})$ [14]. Adams et al. [15] reported that the oil bodies of pumpkin seed proved to be stable to aggregation at $\mathrm{pH}$ values that were sufficiently far from their isoelectric point ( $\mathrm{pH} 3-3.5)$ at relatively low salt concentrations $(<50 \mathrm{mM})$, and they were stable at temperatures to $37.4^{\circ} \mathrm{C}$. In the abovementioned papers, $\zeta$-potential has been widely applied as an essential indicator to evaluate the stability of colloid particles $[14,16-18]$. If the absolute value of $\zeta$-potential of suspending particles was high, it meant these particles were excluded by each other and there was no trend to flocculation [19]. To 
gain more information about the behavior of oil bodies in an aqueous environment, much more properties of oil bodies that originated from other oilseeds should also be conducted.

Peanuts are an important oilseed and economic crop in China. The total output of peanut in China is at the leading position around the world in 2015, which reaches 16,500 thousand metric tons (FAS-USDA) [20]. More than $80 \%$ of production in China takes place in eight provinces: Henan, Shandong, Hebei, Guangdong, Anhui, Sichuan, Liaoning, and Guangxi. Moreover, Henan is also the largest producers among these eight provinces [21]. There are mainly four classes of peanut in Henan: yuhua series, kainong series, puhua series, and yuanza series. Yuhua series grow most widely in Henan province [22]. Peanut is typically characterized by approximately $36-54 \%$ lipids, $21-36 \%$ proteins, and a low percentage of carbohydrates and ash [23]. To our knowledge, however, the stability of oil bodies from a number of sources has been carried out until now; handful of literatures on oil bodies prepared from peanut was source able and studied. And there is much less research on cellular distribution in cells and properties of oil bodies from different peanut varieties under various environmental conditions.

Oil bodies were recovered in the form of cream (oil bodies) using an aqueous extraction method in our work. The characteristic differences of oil bodies that were extracted from different peanut varieties were observed with transmission electron microscopy and a particle size analyzer before and after extraction. The charge stability of oil bodies extracted from five peanut varieties and how they were affected by $\mathrm{pH}$ and ionic strength were also the main goals of this study.

\section{Materials and Methods}

2.1. Materials. Peanuts of five varieties (yuhua23, yuhua27, yuhua9719, yuhua9830, and yuhua9502 harvested in August 2014 in Henan province, China) were supplied by Henan Academy of Agricultural Sciences and stored at $4^{\circ} \mathrm{C}$ until used. The composition ( $\mathrm{g} \mathrm{kg}^{-1}$ dry matter) of peanuts is shown in Table 1. Glutaraldehyde, osmium tetraoxide, Spurr's resin, uranyl acetate, and lead citrate were purchased from SPI Supplies. Other chemicals, which were of analytical-grade, were purchased from Sinopharm Chemical Reagent Co., Ltd. (Shanghai, China). Water was obtained from a Millipore water purification system ( $\geq 18.2 \mathrm{M} \Omega$, Milli-Q, Millipore) and it was used in all runs.

2.2. Analysis of Oil Bodies in the Peanut Cell with Transmission Electron Microscopy. Sections of 1-2 mm of five seeds each per variety, which were cut in the middle transversally by a razor blade, were fixed in $2.5 \%(\mathrm{v} / \mathrm{v})$ glutaraldehyde in sodium phosphate buffer $(0.1 \mathrm{M}, \mathrm{pH} 7.2)$ at $4^{\circ} \mathrm{C}$ overnight and washed for $30 \mathrm{~min}$ with the same buffer three times. Samples were postfixed in $1 \%(\mathrm{w} / \mathrm{v})$ osmium tetraoxide for $2 \mathrm{~h}$, washed $30 \mathrm{~min}$ with the same buffer three times, and then dehydrated in a series of increasing acetone concentrations. Dehydrated samples were infiltrated progressively and embedded in Spurr's resin and cut into ultrathin sections (50-70 $\mathrm{nm}$ thick)
TABLE 1: Composition (g/100 g dry matter) of five peanut varieties.

\begin{tabular}{lccc}
\hline Peanut varieties & Lipid & Protein & Lipid/protein rate \\
\hline Yuhua23 & $45.4 \pm 1.0^{\mathrm{B}}$ & $22.5 \pm 0.3^{\mathrm{B}}$ & 2.0 \\
Yuhua27 & $43.9 \pm 0.8^{\mathrm{C}}$ & $22.0 \pm 0.5^{\mathrm{B}}$ & 2.0 \\
Yuhua9719 & $41.3 \pm 0.8^{\mathrm{D}}$ & $26.5 \pm 0.6^{\mathrm{A}}$ & 1.6 \\
Yuhua9830 & $48.7 \pm 0.9^{\mathrm{A}}$ & $21.1 \pm 0.4^{\mathrm{C}}$ & 2.3 \\
Yuhua9502 & $48.3 \pm 1.0^{\mathrm{A}}$ & $22.5 \pm 0.4^{\mathrm{B}}$ & 2.1 \\
\hline
\end{tabular}

Data were expressed as means \pm standard deviations. The data in the lipid and protein column marked with different capital letters were significantly $(P<0.01)$ different.

with an ultramicrotome (UC5; Leica, Wetzlar, Germany). Sections were mounted on copper grids, stained with $2 \%$ uranyl acetate and Reynold's lead citrate for $10 \mathrm{~min}$ each, and examined with a transmission electron microscope (H-7650; Hitachi Chiyoda-ku, Tokyo, Japan) that was operated at an accelerating voltage of $80 \mathrm{kV}$. Images were recorded with a $4 \mathrm{~K}$ CCD camera (832 ORIUS; Gatan, Pleasanton, CA, USA). Three biological repeats were performed for each peanut variety, and more than 10 ultrathin sections per block were examined [24].

2.3. Size Measurements of Oil Bodies in the Peanut Cell. In order to determine the oil bodies and protein bodies diameter, 80 to 140 measurements of oil body diameter and 15 to 20 measurements of protein body diameter were taken on each image (from 10 images per sample type) obtained from TEM at the same magnification $(5 \mu \mathrm{m}$ or $6000 \mathrm{x})$ by Nano Measurer 1.2. The determination of mean diameter of the oil bodies and protein bodies was based on the results of 10 images. Statistical analysis of the data was performed using the SPSS 19.0 software.

2.4. Extraction of Oil Bodies from Peanut Seeds. Oil bodies were isolated using an aqueous extraction method with some modifications $[8,25]$. In brief, the dehulled peanut seeds were peeled and then were comminuted by liquid nitrogen. A $20 \mathrm{~g}$ sample of crushed peanuts was transferred to the beaker and dispersed in deionised water at a 1:5 (wt/vol) seeds-towater ratio. The mixture was stirred by a Fluko homogenizer (18 $000 \mathrm{rpm}, \mathrm{FM} 200)$ for $10 \mathrm{~s}$. The mixture was then incubated for $1 \mathrm{~h}$ at $45^{\circ} \mathrm{C}$ in a constant temperature bath shaker (THZ82, Huafeng, Jintan, China). Then, the resulting slurry was filtered through four layers of gauze cloth. The filtrate was handled with a centrifuge (DZ267-32C6, anting, Shanghai, China) at $4000 \mathrm{rpm}$ for $30 \mathrm{~min}$. The oil bodies appeared as a cream at the top of the centrifuge tube, which was collected in a tube as a creamy pad from the top of the mixture. Oil bodies were stored at $4^{\circ} \mathrm{C}$ for not more than $24 \mathrm{~h}$.

2.5. Particle Size Analysis of Peanut Oil Bodies after Aqueous Extraction. Oil bodies were diluted to a concentration of approximately $0.1 \mathrm{wt}$./vol by adding water to the beaker, and then it was leached by ultrasonic baths to ensure homogeneity. The particle diameter distribution was measured using a laser particle size analyzer (Microtrac S3500, America). 
2.6. Preparation of Oil Body Suspensions and Measurements of Zeta $(\zeta)$ Potential. The influence of $\mathrm{pH}$ and ionic strength on oil body suspensions was achieved by the method [15], with slight modifications. Oil body suspensions were made by suspending $1 \mathrm{~g}$ of cream with $9 \mathrm{~g}$ of buffer solution $10 \mathrm{mM}$ $\mathrm{Na}_{2} \mathrm{HPO}_{4} \cdot \mathrm{NaCl}(0,10,40,80$, and $100 \mathrm{mM})$ at $\mathrm{pH} 3.0,7.4$, and 9.0, which was then incubated at $37^{\circ} \mathrm{C}$ for $20 \mathrm{~min}$ and stored at the room temperature $\left(25^{\circ} \mathrm{C}\right)$ for $24 \mathrm{~h}$ prior to the analysis of $\zeta$-potential.

Suspensions obtained from the treatments above were diluted to a concentration of approximately $0.05 \mathrm{wt}$./vol. Diluted suspensions were measured directly by a zeta $(\zeta$ ) potential analyzer (Brookhaven Instruments Corporation, America). $\zeta$-potential of the emulsion was measured three times with two freshly prepared parallel samples. Statistical analysis of the data was performed using the SPSS 19.0 software.

\section{Results and Discussion}

3.1. Size of Oil Body from Five Peanut Varieties in the Cell. In china, Henan ranks as the Chinese largest peanut producer, which is located in the Yellow River basin. There are mainly four classes of peanut in Henan: yuhua series, kainong series, puhua series, and yuanza series. Meanwhile, yuhua series grow most widely in Henan province [22]. In this work, the common varieties (yuhua23; yuhua27; yuhua9502; yuhua9719; and yuhua9830) in the yuhua series were selected in order to reflect some difference of oil body properties between peanut varieties basing the difference of lipid and protein content. The average values of lipid and protein content of these varieties are shown in Table 1. The results showed that the protein ranged from $211 \mathrm{~g} \mathrm{~kg}^{-1}$ to $265 \mathrm{~g} \mathrm{~kg}^{-1}$, and the lipid varied from $413 \mathrm{~g} \mathrm{~kg}^{-1}$ to $487 \mathrm{~g} \mathrm{~kg}^{-1}$. Normally, Henan peanuts are characterized by approximately $440-$ $560 \mathrm{~g} \mathrm{~kg}^{-1}$ lipid and 220-300 $\mathrm{g} \mathrm{kg}^{-1}$ protein [22]. Table 1 also shows that the five peanut varieties involved high, middle, and low levels of lipid and protein content. All data in the Table 1 were processed by SPSS; the results of mono factor analysis of variance indicated that there existed obvious difference of lipid and protein content $(P<0.01)$ between peanut varieties.

The original oil bodies that were distributed throughout peanut cells were generally sphere-shaped (Figure 1). These entities were abundant, and they occupied much more intracellular volume than the protein bodies that were surrounded by oil bodies. Oil bodies and protein bodies were closely associated in the peanut cells. This distribution is different than in soybean cotyledons [26]. As shown in Figure 1, there was no uniformity in size, distribution, or organization of oil bodies among the different peanut varieties. Moreover, the morphological characteristics of oil bodies and protein bodies of peanuts showed that the oil body and protein body were closely inside the cells and this appearance might determine the interaction of oil and protein in extraction. Moreover, in this case, oil bodies could maintain their spherical shape, even if sometimes they appear to be pressed against each other [11]. Measurements of oil bodies and protein bodies diameter, where possible, revealed an average diameter from five peanut varieties (Table 2). The mean diameter of oil bodies from yuhua9719 and yuhua9830 is apparently larger than yuhua23, yuhua27, and yuhua9502 $(P<0.01)$. Yuhua27 had much larger protein bodies than the other four peanut varieties (Table 2). Additionally, the standard value of deviation of oil bodies of yuhua27 and protein bodies of yuhua 9719 was smaller than the other varieties (Table 2), so yuhua27 and yuhua 9719 had a more uniform size of oil body and protein body, respectively. Oil body diameter range of all examined samples is calculated from Table 2; yuhua27 shows a narrower distribution range of $0.74-2.83 \mu \mathrm{m}$. For another thing, the wider diameter range of protein body from five peanut varieties is yuhua27 $(2.52-13.49 \mu \mathrm{m})$. It was indicated that there existed an intraspecific difference in size of oil body and protein body [27]. These characteristics may partially correlate with the content of oil and protein. For example, oil bodies of strains of maize with high oil content were larger and more regular compared with a strain with low oil content, although these two strains were characterized by different morphology [28]. However, there emerged a case that yuhua9719 with low oil and high protein content also had a larger oil body mean diameter. So, it was possible that larger diameter of oil bodies was due to the lower or higher rate of oil and protein content, which was not consistent with the results from previous research [11]. All these differences in distribution and size characteristics among the five peanut varieties were greatly attributed to differences in genotypes, given that the analyzed varieties had the same geographical origin and were grown under similar agronomic conditions. These observations reflected the possibility that there is intraspecies morphological variation among oil bodies, as suggested by Crisafulli et al. [29].

\subsection{Size Change of Oil Bodies from Five Peanut Varieties} after Aqueous Extraction. To understand the difference and change in size of oil body further, the median diameter of peanut oil bodies from different varieties was determined after aqueous extraction (Figure 2). Yuhua27 and yuhua9502 showed a narrow distribution, with median diameter (D50) of $4.08 \mu \mathrm{m}$ and $3.01 \mu \mathrm{m}$, respectively. Yuhua9719 and yuhua9830 exhibited a similar distribution, with about $50 \%$ of oil bodies less than $13.86 \mu \mathrm{m}$ and $13.03 \mu \mathrm{m}$, respectively. The D50 of peanut variety yuhua23 was $7.89 \mu \mathrm{m}$. These results showed that the mean diameter of oil bodies clearly became enlarged after extraction compared with the initial state in the cell (Table 2). Similar results were obtained where the median diameters of oil bodies from yuhua9719 and yuhua9830 were obviously larger than the other peanut varieties compared with the initial diameter in the peanut cell (see Table 2). The reason may be that the protein in the peanut was released, moved to the surface of the oil body, and exposed buried hydrophobic amino acids to the surface of the oil body during extraction [30]. Consequently, the initial oil bodies and the coextracted exogenous proteins (storage proteins) probably formed a second layer around the oil bodies [5]. Thus, the diameters of extracted oil bodies were larger than the initial size in the peanut cell. 
TABLE 2: Oil bodies and protein bodies diameter (mean \pm standard deviation) and minimum $(d \mathrm{~min})$ and maximum diameter $(d \mathrm{max})(\mu \mathrm{m})$.

\begin{tabular}{|c|c|c|c|c|c|c|}
\hline \multirow{2}{*}{ Peanut varieties } & \multicolumn{3}{|c|}{ Oil bodies } & \multicolumn{3}{|c|}{ Protein bodies } \\
\hline & Mean diameter & $d \min$ & $d \max$ & Mean diameter & $d \min$ & $d \max$ \\
\hline Yuhua23 & $1.59 \pm 0.09^{\mathrm{B}}$ & 0.71 & 3.00 & $4.52 \pm 0.72^{\mathrm{B}}$ & 1.62 & 9.53 \\
\hline Yuhua27 & $1.63 \pm 0.17^{\mathrm{B}}$ & 0.74 & 2.83 & $7.88 \pm 4.00^{\mathrm{A}}$ & 2.52 & 13.49 \\
\hline Yuhua9719 & $1.94 \pm 0.29^{\mathrm{A}}$ & 0.61 & 3.96 & $3.30 \pm 0.61^{\text {B }}$ & 1.05 & 6.40 \\
\hline Yuhua9830 & $1.92 \pm 0.19^{\mathrm{A}}$ & 0.87 & 5.04 & $3.73 \pm 0.65^{\mathrm{B}}$ & 1.46 & 6.95 \\
\hline Yuhua9502 & $1.65 \pm 0.15^{\mathrm{B}}$ & 0.55 & 3.16 & $2.75 \pm 0.95^{\mathrm{B}}$ & 0.87 & 6.02 \\
\hline
\end{tabular}

Values are means $\pm \mathrm{SD}$, with the $P$ value $<0.01$ from a one-way ANOVA analysis. Letters after means indicate significant differences in means.

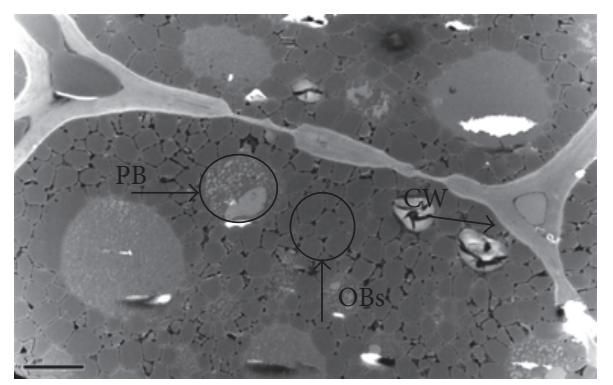

(a)

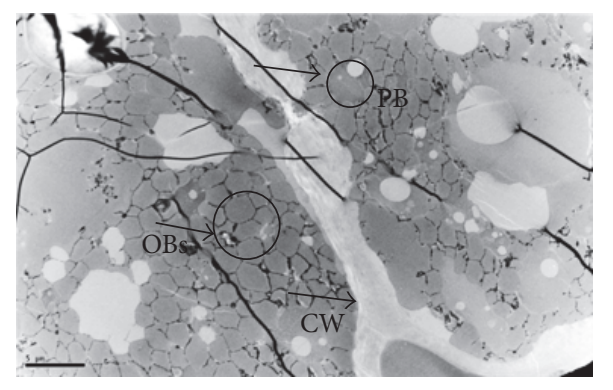

(c)

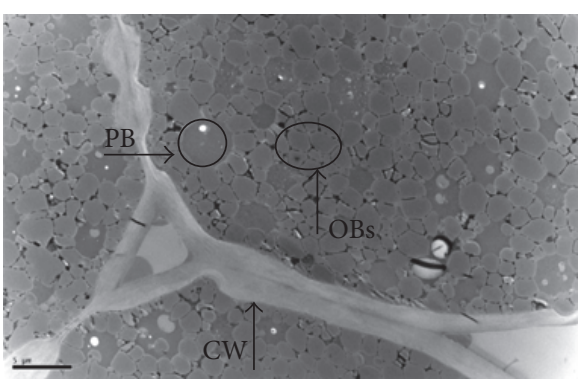

(b)

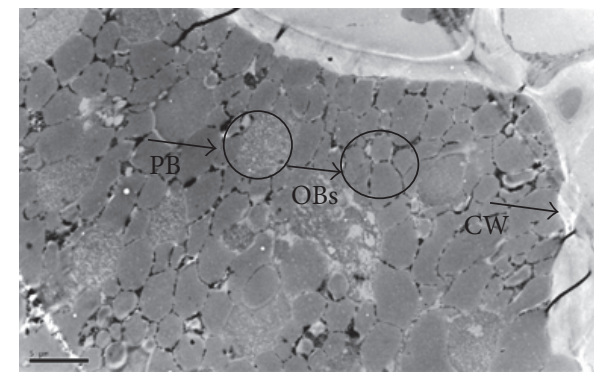

(d)

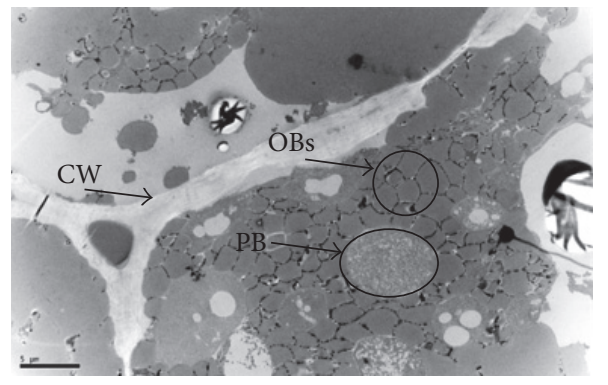

(e)

FIGURE 1: The transmission electron microscope (TEM) pictures of five peanut varieties ((a) yuhua23, (b) yuhua27, (c) yuhua9719, (d) yuhua9830, and (e) yuhua9502). Bar represents $5 \mu \mathrm{m}$ (PB, protein body; CW, cell wall; OBs, oil bodies).

In addition, the degree of difference among the diameter of oil bodies was different, which was yuhua9719 > yuhua9830 $>$ yuhua23 > yuhua27 > yuhua9502; this sequence is almost in accordance with mean diameter level of peanut oil bodies in cell. However, the degree of increase in diameter was various between peanut varieties. These differences may have been due to the different amounts of acidic amino acid and protein structure on the surface of the oil bodies among the different peanut varieties [31]. For instance, yuhua9719 and yuhua9830 showed a much wider diameter distribution than yuhua27 and yuhua9502, because yuhua9719 contained much more protein, and yuhua9830 contained much more oil. That is, much more protein was attached to oil bodies in yuhua9719, but many more oil bodies potentially aggregated to form still larger units for $y$ uhua 9830 in the extraction. The distributions of protein bodies and oil bodies, the contents of protein and oil, and the ratio of protein and oil may combine to have a joint effect on the diameters of oil bodies in aqueous 


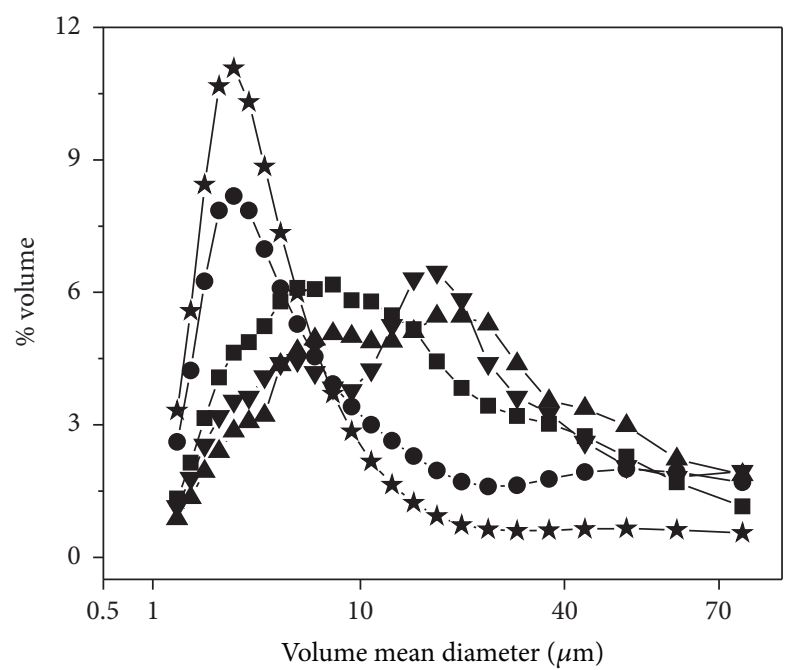

Figure 2: Diameter distribution of oil bodies extracted from different peanut varieties (ロ yuhua23, - yuhua27, \ yuhua9719, $\boldsymbol{\nabla}$ yuhua9830, and $\star$ yuhua9502). Results are expressed as the percentage of the total volume of all the lipid particles.

extraction [3]. When exogenous protein reaches the surface of oil bodies, strong viscoelastic films can be developed that resist environmental stresses and which provide electrostatic and steric stabilization [32]. Thus, understanding the charge stability of oil bodies that are extracted from different peanut varieties may provide important insights into the stability of peanut oil bodies and it might also yield guidelines as to the type of food matrices (e.g., acidic, neutral, and alkaline) in which oil bodies of suitable peanut varieties could be utilized successfully.

3.3. Influence of pH on Charge Stability of Oil Bodies Extracted from Five Peanut Varieties. Zeta $(\zeta)$ potentials could be used as indicators of the stability of oil body suspensions [16]. Guidelines classifying particles dispersions with $\zeta$-potential values of $\pm(0-10) \mathrm{mV}, \pm(10-20) \mathrm{mV}$, and $\pm(20-30) \mathrm{mV}$ and $> \pm 30 \mathrm{mV}$ as highly unstable, relatively stable, moderately stable, and highly stable [33]. $\zeta$-potentials of the oil body suspensions (in $10 \mathrm{mM}$ phosphate buffer) in different salt concentrations $(0-100 \mathrm{mM})$ were observed at various $\mathrm{pH}$ values (pH3.0, pH7.4, and pH9.0), with the aim of knowing how charge stability would be affected by a combination of $\mathrm{pH}$ and salt concentration (Figure 3). The results of tests of betweensubjects effects of the univariate analyses concerning $\zeta$ potentials of oil bodies extracted from different peanut varieties at different salt concentrations under various $\mathrm{pH}$ are also given by SPSS. It indicated that $\mathrm{NaCl}$ concentration $(P<0.001)$ and $\mathrm{pH}(P<0.001)$ were significantly associated with $\zeta$-potential. Additionally, we modeled the impact of the variables jointly and detected that interaction of $\mathrm{pH}$ and $\mathrm{NaCl}$ concentration was also significant $(P<0.001)$.

$\zeta$-potentials of the oil bodies extracted from five peanut varieties remained positive at pH3.0 (Figure 3(a)). On the other hand, these values remained negative at pH7.4 and pH9.0 (Figures 3(b)-3(c)). The better charge stability of these five peanut varieties was at $\mathrm{pH} 7.4$ without salt. Cao et al. [34] proved that the charge property of oil bodies was affected by $\mathrm{pH}$, possibly because amino acid residues at the $\mathrm{N}$ - and $\mathrm{C}$ terminus of the surface of oil bodies are attributed to $\mathrm{pH}$. The positively charged amino acid residues of the $\mathrm{N}$ - and $\mathrm{C}$ terminus are deprotonated by alkaline $\mathrm{pH}$, which decreases the number of salt bridges between oleosins and phospholipids. As a result, contaminated proteins could not anchor to oil bodies as strongly as before [34], and the coalescence of oil bodies was more likely to emerge. Consequently, oil bodies have a net positive charge under acidic conditions and a net negative charge in the neutral or alkaline environment. In this experiment, five peanut varieties had different charge levels at the same $\mathrm{NaCl}$ concentration under $\mathrm{pH} 3.0,7.4$, and 9.0, which may be due to differences in the amount of acidic amino acid in the protein structure on the surface of the oil bodies [31]. For instance, two rapeseed genotypes (Amber and Warzanwski) have different stability due to the different composition of $\mathrm{H}$-oleosins and steroleosins, phospholipids, and sterols on OBs surface [12].

With the increase in $\mathrm{pH}, \zeta$-potential of oil bodies changed from positive to negative in accordance with the surface charge under various salt concentrations. In this test, the isoelectric point of oil bodies was at $\zeta$-potential of zero (these figures not show). Oil body suspensions have maximal destabilization at these zero $\zeta$-potentials and this property can be used as an estimate of the isoelectric point [25]. The isoelectric point of oil bodies from different peanut varieties was predicted according to $\zeta$-potential of pH3.0 and pH7.4 under different conditions of ion strength $(\mathrm{NaCl}$ concentration of $0,10,40,80$, and $100 \mathrm{mM}$ ). The isoelectric point of oil bodies from yuhua23, yuhua27, yuhua9719, yuhua9830, and yuhua 9502 was between pH4.5 and pH5.5 at $0-10 \mathrm{mM} \mathrm{NaCl}$ concentrations, and our isoelectric points were distinctly different from some published results $[4,7,14,35,36]$. With the further increase in $\mathrm{NaCl}$ concentration, the point of zero charge of five peanut varieties changed. Therefore, further work is required to establish the potential role of the point of zero charge on the stability of oil bodies extracted from different peanut varieties.

\subsection{Influence of Salt Concentration on Charge Stability of Oil} Bodies Extracted from Five Peanut Varieties. With increasing $\mathrm{NaCl}$ concentration at different $\mathrm{pH}$ values, $\zeta$-potentials of oil bodies from all five peanut varieties fell significantly (Figures 3(a)-3(c)). High salt concentration resulted in a distinctly lower stability at $\mathrm{pH} 3.0, \mathrm{pH} 7.4$, and $\mathrm{pH} 9.0$. At $\mathrm{pH} 3.0, \zeta$ potential of oil bodies was over $22 \mathrm{mV}$ in the absence of salt, which indicated that moderate charge stability exists. As the salt concentration increased, the positive value declined. $\zeta$ potential of yuhua27 and yuhua9719 fell ultimately by $72 \%$ and $73 \%$, respectively, but $\zeta$-potential of yuhua9830 and yuhua9502 decreased only slightly. However, the absolute value of $\zeta$-potentials of yuhua9502 and yuhua9719 declined dramatically by $92 \%$ and $90 \%$ at $\mathrm{pH} 7.4$ in the given range of $0-100 \mathrm{mM} \mathrm{NaCl}$ concentrations. The top $\zeta$-potential value of these peanut varieties appears under the condition of $0 \mathrm{mM}$ $\mathrm{NaCl}$ at pH7.4. Particularly, $\zeta$-potentials of yuhua27 and 

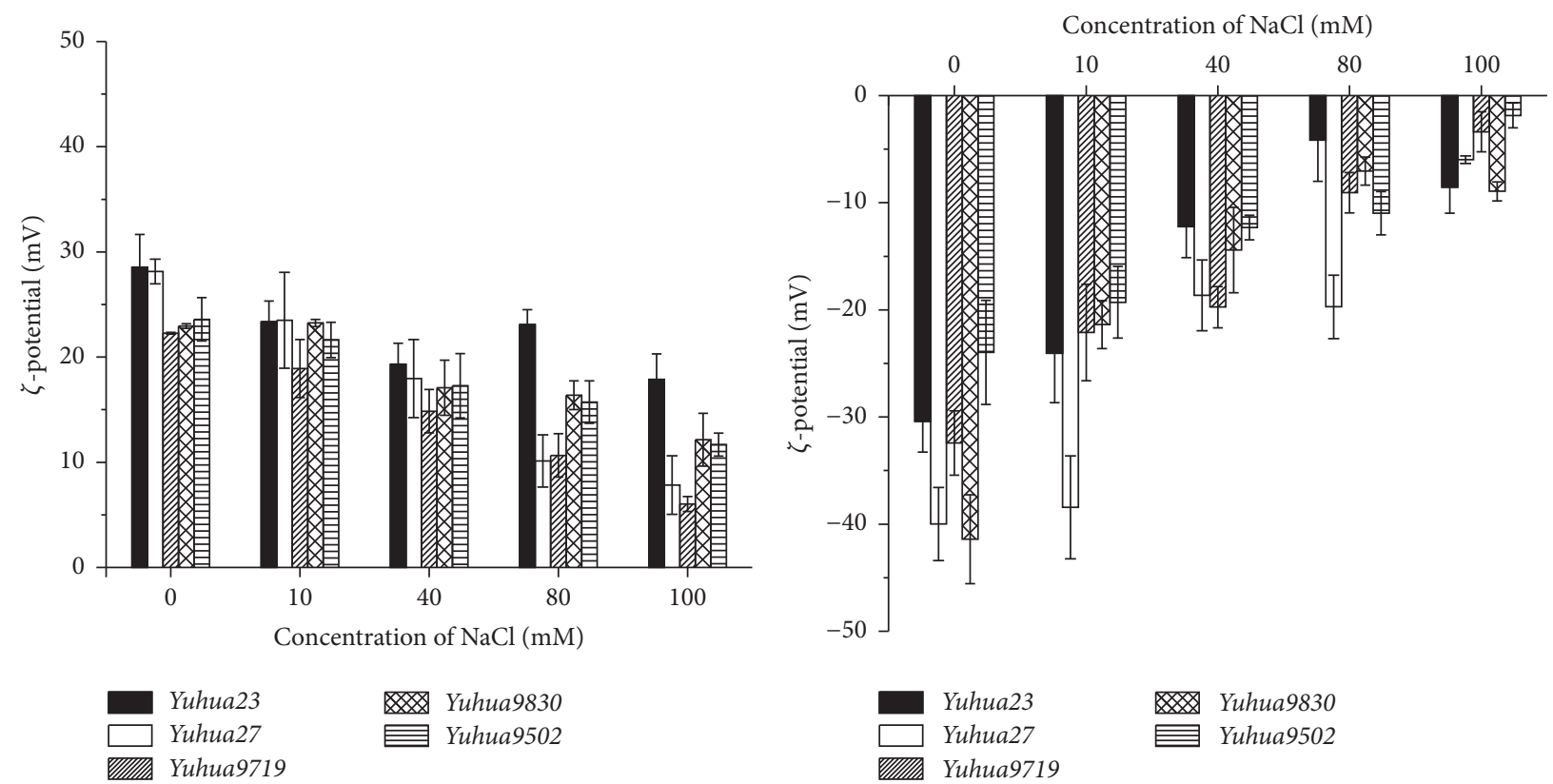

(a)

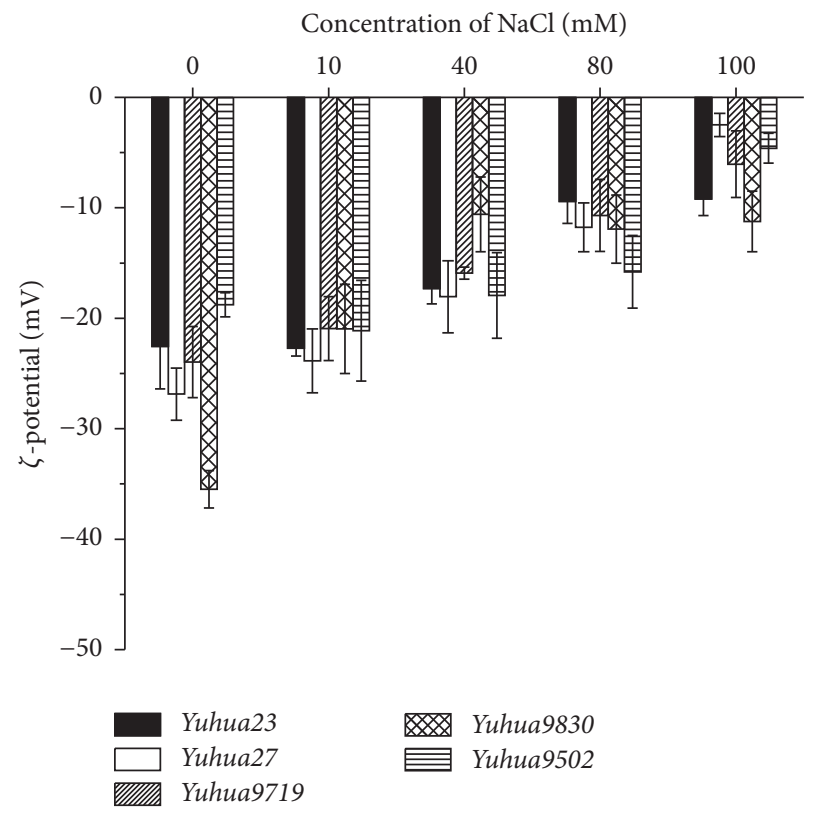

(c)

Figure 3: $\zeta$-potential of peanut oil bodies at different salt concentrations under various $\mathrm{pH}$ values ((a) $\mathrm{pH} 3.0,(\mathrm{~b}) \mathrm{pH}$.4, and (c) $\mathrm{pH}$ 9.0), where error bar represents standard deviation.

yuhua9830 were as high as $-40 \mathrm{mV}$. At $\mathrm{pH} 9.0$, the absolute value was significantly lower for yuhua27 and yuhua9719 at $0-100 \mathrm{mM} \mathrm{NaCl}$ concentrations, but $\zeta$-potential of yuhua23 was reduced only slightly.

These results showed that there was a general decrease in the absolute value of $\zeta$-potential with increasing $\mathrm{NaCl}$ concentration, and that all five peanut varieties were stable $(\zeta$-potential $>20 \mathrm{mV})$ at low salt concentrations $(<10 \mathrm{mM})$ at pH7.4 and pH9.0. Meanwhile, yuhua27 and yuhua9830 exhibited outstanding charge stability $(\zeta$-potential $>35 \mathrm{mV})$ in neutral microenvironment without salt concentration. In particular, yuhua9830 had nice charge stability in neutral and alkaline microenvironment without salt concentration. Thus, the adaptability of oil bodies from yuhua9830 to neutral and alkaline condition was better than other four peanut varieties. Similar results were obtained in soybean oil bodies that were uncoated by $\kappa$-carrageenan [37] and in oil bodies from a maize germ suspension in the absence of SDS [16]. This decrease in $\zeta$-potential absolute value in the range of $0-100 \mathrm{mM} \mathrm{NaCl}$ concentrations may be due to electrostatic 
screening effects and to decreases in the energy of the electrostatic interaction brought about by the salt $[38,39]$. The relative insensitivity of $\zeta$-potential of oil bodies from some peanut varieties to the addition of $\mathrm{NaCl}$ may be due to the presence of some endogenous salt in the peanut cell [14]. Thus, low salt concentration can benefit the solubility of the surface protein of oil bodies in the aqueous media and enhance the stability of oil bodies, although increased salt concentrations have the opposite effect on the stability for the weaken protein hydration [14].

\section{Conclusion}

With the aim of understanding the possible difference of oil bodies from different varieties, the diameter and zeta $(\zeta)$ potential of oil bodies from five peanut varieties ( $y$ uhua23, yuhua27, yuhua9719, yuhua9830, and yuhua9502) were studied. The results showed that oil bodies occupied much more intracellular volume than the protein bodies that were surrounded by oil bodies. The differences in size and distribution of oil bodies and protein bodies in peanut cells existed among five peanut varieties. In the peanut cell, the mean diameters of oil bodies from yuhua9719 and yuhua9830 were apparently larger than yuhua23, yuhua27, and yuhua9502; yuhua27 had much larger protein bodies than the other four peanut varieties. The median diameter of oil bodies from five peanut varieties increased markedly and variously compared with the initial oil bodies in a cell after aqueous extraction, and the degree of increase is based on the diameter level of oil bodies in cell. The zeta $(\zeta)$ potential results of oil bodies from five peanut varieties under different conditions of $\mathrm{pH}$ and salt concentration showed that oil bodies were stable at low salt concentrations $(<10 \mathrm{mM})$ at pH7.4 and pH9.0 at room temperature. Of the five peanut varieties, yuhua27 and yuhua9830 possessed excellent charge stability $(\zeta$-potential $>35 \mathrm{mV})$ in neutral microenvironment without salt concentration. In particular, the adaptability of oil bodies from yuhua9830 to neutral and alkaline condition was better than other four peanut varieties.

\section{Disclosure}

This article does not contain any studies with human or animal subjects.

\section{Competing Interests}

The authors declare that there are no competing interests regrading this paper.

\section{Acknowledgments}

This work was supported by National Science Foundation of China (no. 21376064 and no. 21176058).

\section{References}

[1] J. T. C. Tzen, "Integral proteins in plant oil bodies," ISRN Botany, vol. 2012, Article ID 173954, 16 pages, 2012.

[2] S. Gallier, K. C. Gordon, and H. Singh, "Chemical and structural characterisation of almond oil bodies and bovine milk fat globules," Food Chemistry, vol. 132, no. 4, pp. 1996-2006, 2012.

[3] C. V. Nikiforidis, A. Matsakidou, and V. Kiosseoglou, "Composition, properties and potential food applications of natural emulsions and cream materials based on oil bodies," RSC Advances, vol. 4, no. 48, pp. 25067-25078, 2014.

[4] C. V. Nikiforidis and V. Kiosseoglou, "Aqueous extraction of oil bodies from maize germ (Zea mays) and characterization of the resulting natural oil-in-water emulsion," Journal of Agricultural and Food Chemistry, vol. 57, no. 12, pp. 5591-5596, 2009.

[5] C. V. Nikiforidis, V. Kiosseoglou, and E. Scholten, "Oil bodies: an insight on their microstructure-maize germ vs sunflower seed," Food Research International, vol. 52, no. 1, pp. 136-141, 2013.

[6] A. H. C. Huang, "Oleosins and oil bodies in seeds and other organs," Plant Physiology, vol. 110, no. 4, pp. 1055-1061, 1996.

[7] J. T. C. Tzen, Y.-Z. Cao, P. Laurent, C. Ratnayake, and A. H. C. Huang, "Lipids, proteins, and structure of seed oil bodies from diverse species," Plant Physiology, vol. 101, no. 1, pp. 267-276, 1993.

[8] C. V. Nikiforidis, O. A. Karkani, and V. Kiosseoglou, "Exploitation of maize germ for the preparation of a stable oilbody nanoemulsion using a combined aqueous extractionultrafiltration method," Food Hydrocolloids, vol. 25, no. 5, pp. 1122-1127, 2011.

[9] G. I. Frandsen, J. Mundy, and J. T. C. Tzen, "Oil bodies and their associated proteins, oleosin and caleosin," Physiologia Plantarum, vol. 112, no. 3, pp. 301-307, 2001.

[10] A. H. C. Huang, "Oil bodies and oleosins in seeds," Annual Review of Plant Physiology and Plant Molecular Biology, vol. 43, no. 1, pp. 177-200, 1992.

[11] P. Crisafulli, L. Navarini, F. Silizio, A. Pallavicini, and A. Illy, "Ultrastructural characterization of oil bodies in different coffea species," Tropical Plant Biology, vol. 7, no. 1, pp. 1-12, 2014.

[12] C. Boulard, M. Bardet, T. Chardot et al., "The structural organization of seed oil bodies could explain the contrasted oil extractability observed in two rapeseed genotypes," Planta, vol. 242, no. 1, pp. 53-68, 2015.

[13] P. Jolivet, C. Deruyffelaere, C. Boulard et al., "Deciphering the structural organization of the oil bodies in the Brassica napus seed as a mean to improve the oil extraction yield," Industrial Crops and Products, vol. 44, pp. 549-557, 2013.

[14] D. Iwanaga, D. A. Gray, I. D. Fisk, E. A. Decker, J. Weiss, and D. J. McClements, "Extraction and characterization of oil bodies from soy beans: a natural source of pre-emulsified soybean oil," Journal of Agricultural and Food Chemistry, vol. 55, no. 21, pp. 8711-8716, 2007.

[15] G. G. Adams, S. Imran, S. Wang et al., "Extraction, isolation and characterisation of oil bodies from pumpkin seeds for therapeutic use," Food Chemistry, vol. 134, no. 4, pp. 1919-1925, 2012.

[16] R. Sukhotu, X. Shi, Q. Hu, K. Nishinari, Y. Fang, and S. Guo, "Aggregation behaviour and stability of maize germ oil body suspension," Food Chemistry, vol. 164, no. 12, pp. 1-6, 2014.

[17] N. Nantiyakul, S. Furse, I. D. Fisk, G. Tucker, and D. A. Gray, "Isolation and characterization of oil bodies from Oryza sativa 
bran and studies of their physical properties," Journal of Cereal Science, vol. 57, no. 1, pp. 141-145, 2013.

[18] X. Shi and S. Guo, "Effect of diluent type on analysis of zeta potential of colloid particles of soymilk protein," Transactions of the Chinese Society of Agricultural Engineering, vol. 32, no. 7, pp. 270-275, 2016.

[19] M. Kosmulski, "Zeta potentials in nonaqueous media: how to measure and control them," Colloids and Surfaces A: Physicochemical and Engineering Aspects, vol. 159, no. 2-3, pp. 277-281, 1999.

[20] FAS-USDA.Oilseeds: World Markets and Trade, http://apps.fas .usda.gov/psdonline/psdHome.aspx.

[21] W. M. Zhang, Rural Statistical Yearbook in China, China Statistics Press, Beijing, China, 2015 (Chinese).

[22] S. L. Yu, Chinese Peanut Varieties and Their Genealogy, Shanghai scientific and Technical Publishers, Shanghai, China, 2008 (Chinese).

[23] C. L. Hoffpauir, "Peanut composition: relation to processing and utilization," Agricultural and Food Chemistry, vol. 1, no. 10, pp. 668-671, 1953.

[24] J. Cao, Y. Song, H. Wu, L. Qin, L. Hu, and R. Hao, "Ultrastructural studies on the natural leaf senescence of Cinnamomum camphora," Scanning, vol. 35, no. 5, pp. 336-343, 2013.

[25] G. Payne, M. Lad, T. Foster, A. Khosla, and D. Gray, "Composition and properties of the surface of oil bodies recovered from Echium plantagineum," Colloids and Surfaces B: Biointerfaces, vol. 116, pp. 88-92, 2014.

[26] A. Rosenthal, D. L. Pyle, and K. Niranjan, "Aqueous and enzymatic processes for edible oil extraction," Enzyme and Microbial Technology, vol. 19, no. 6, pp. 402-420, 1996.

[27] A. Huang, J. Tzen, K. Lee, F. Bih, J. Ting, and C. Ratnayake, "Seed oil bodies in maize and other species," Botanical Bulletin of Academia Sinica, vol. 34, no. 4, pp. 289-297, 1993.

[28] J. T. L. Ting, K. Lee, C. Ratnayake, K. A. Platt, R. A. Balsamo, and A. H. C. Huang, "Oleosin genes in maize kernels having diverse oil contents are constitutively expressed independent of oil contents: size and shape of intracellular oil bodies are determined by the oleosins/oils ratio," Planta, vol. 199, no. 1, pp. 158-165, 1996.

[29] P. Crisafulli, F. Silizio, A. Pallavicini, and L. Navarini, “Oil bodies ultrastructure in green coffee seeds with different geographical origin," in Proceedings of the ASIC 23rd International Conference on Coffee Science, Bali, Indonesia, October 2010.

[30] P. Walstra, Physical Chemistry of Foods, CRC Press, 2002.

[31] L. Wang, Q. Wang, H. Liu, L. Liu, and Y. Du, "Determining the contents of protein and amino acids in peanuts using nearinfrared reflectance spectroscopy," Journal of the Science of Food and Agriculture, vol. 93, no. 1, pp. 118-124, 2013.

[32] S. Tcholakova, N. D. Denkov, I. B. Ivanov, and B. Campbell, "Coalescence stability of emulsions containing globular milk proteins," Advances in Colloid and Interface Science, vol. 123-126, pp. 259-293, 2006.

[33] V. R. Patel and Y. K. Agrawal, "Nanosuspension: an approach to enhance solubility of drugs," Journal of Advanced Pharmaceutical Technology \& Research, vol. 2, no. 2, pp. 81-87, 2011.

[34] Y. Cao, L. Zhao, Y. Ying, X. Kong, Y. Hua, and Y. Chen, "The characterization of soybean oil body integral oleosin isoforms and the effects of alkaline $\mathrm{pH}$ on them," Food Chemistry, vol. 177, pp. 288-294, 2015.

[35] P. C. Calder and P. Yaqoob, "Lipid rafts-composition, characterization, and controversies," The Journal of Nutrition, vol. 137, no. 3, pp. 545-547, 2007.
[36] R. L. C. Chuang, J. C. F. Chen, J. Chu, and J. T. C. Tzen, "Characterization of seed oil bodies and their surface oleosin isoforms from rice embryos," Journal of Biochemistry, vol. 120, no. 1, pp. 74-81, 1996.

[37] N.-N. Wu, X. Huang, X.-Q. Yang et al., "Stabilization of soybean oil body emulsions using $\iota$-carrageenan: effects of salt, thermal treatment and freeze-thaw cycling," Food Hydrocolloids, vol. 28, no. 1, pp. 110-120, 2012.

[38] D. J. McClements, Food Emulsions: Principles, Practices, and Techniques, CRC Press, Boca Raton, Fla, USA, 2005.

[39] G. A. Martínez-Muñoz and P. Kane, "Vacuolar and plasma membrane proton pumps collaborate to achieve cytosolic $\mathrm{pH}$ homeostasis in yeast," Journal of Biological Chemistry, vol. 283, no. 29, pp. 20309-20319, 2008. 

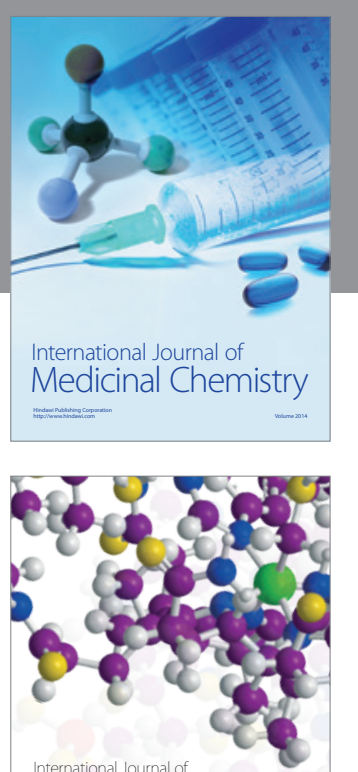

Carbohydrate Chemistry

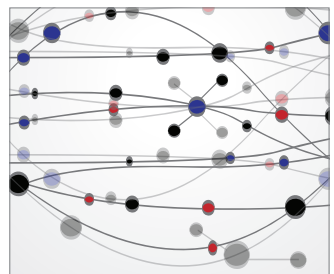

The Scientific World Journal
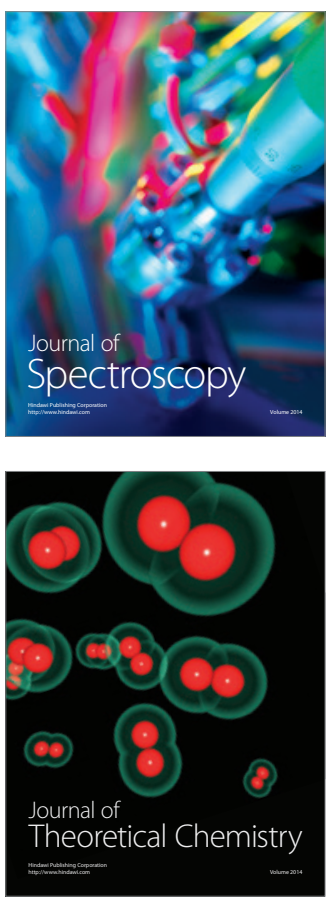
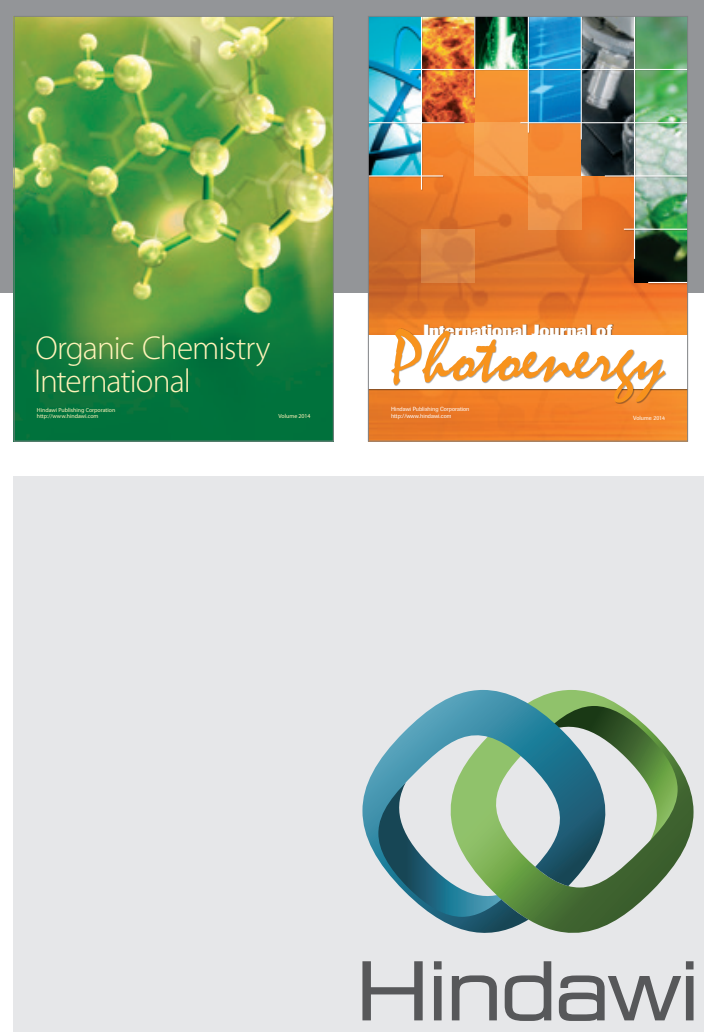

Submit your manuscripts at

http://www.hindawi.com

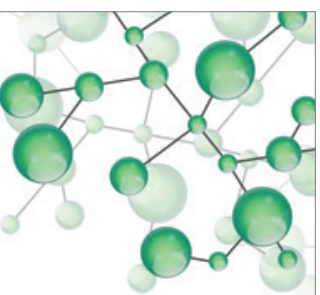

International Journal of

Inorganic Chemistry

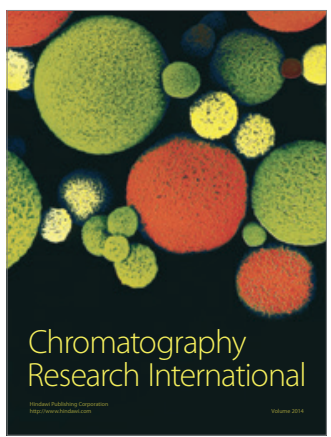

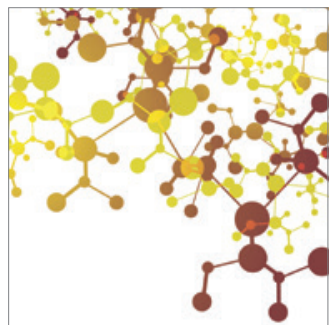

Applied Chemistry
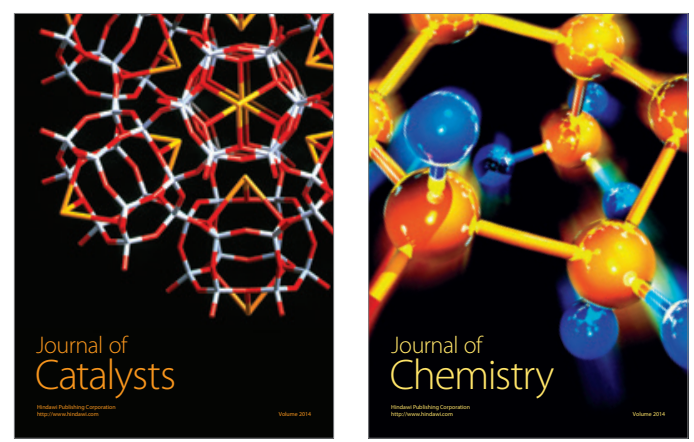
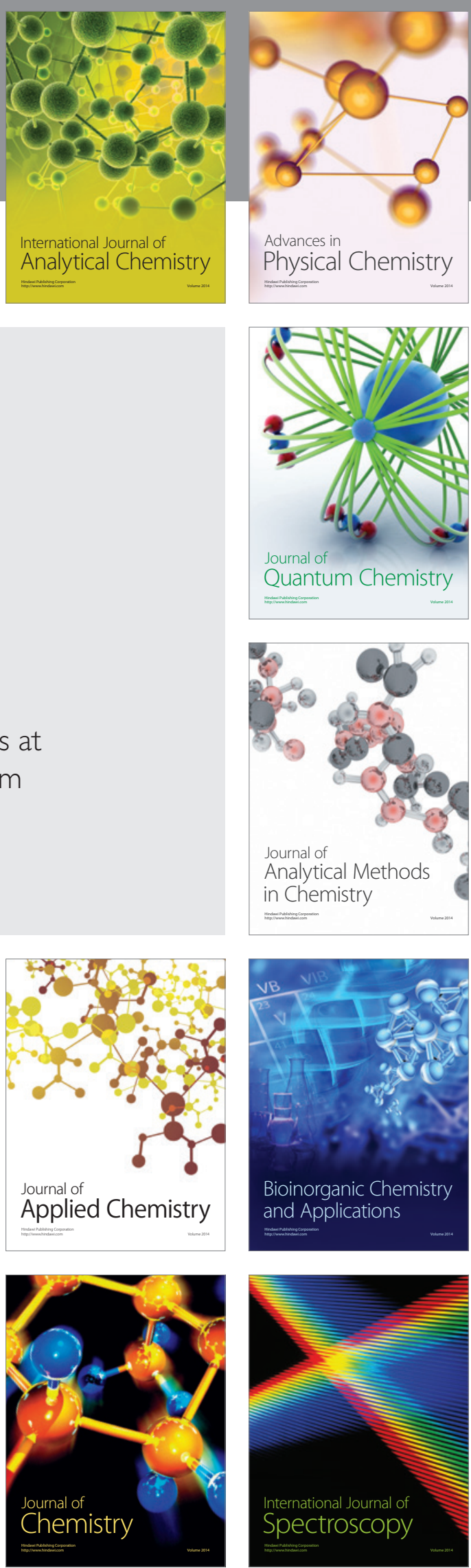